\title{
Demystified...
}

\section{Adhesion molecules}

\author{
A J Freemont
}

\section{Reactions with environments}

Cell adhesion molecules are beginning to play an increasing role in our understanding of disease processes and they are impinging on diagnostic and prognostic histopathology practice. To understand why they are so important to histopathologists necessitates a review of how cells regulate, and are regulated by, their local environment.

THE PROFESSOR IN ITS ENVIRONMENT-A PARADIGM FOR UNDERSTANDING THE CELL Sometimes as I wander around the university I see a colleague and shout a word of greeting; increasingly rarely, I attend NHS management meetings and hear good news; sometimes, when I have been working at the microscope for a few hours, I cannot touch the casing because it is so hot. In every instance I am communicating with my environment and I recognise the signals coming from it using my body surface, my eyes, my ears, and my skin. This interaction with my environment results in a response that comes from far below the surface: the complexities of thought, speech, emotion, or movement. Cells are no different; they also interact with their environments at the level of their surfaces and the result of that interaction is a fundamental change in their biology, often involving complex biochemical events initiated deep within their cytoplasm or nucleus.

RECEPTORS ARE EVERYTHING

Had I no eyes or ears or sensory organs in my skin I would not be able to respond to the signals in my environment. I would walk past my colleague, I would not hear the good news, my hand would remain resting on the microscope casing. This would not be because the signals were no longer present within the environment, on the contrary, nothing would have changed outside my body. The difference would be my ability to recognise the changes going on around me. Cells are just the same. They require response elements on their surfaces with the ability to recognise the changes occurring in their environment if they are to react to those changes.

Therefore, for a cell to respond to a change in its environment, it needs a mechanism to recognise that change.

THE ENVIRONMENT OF THE CELL

The environment of any cell in the body is a very complex mixture of soluble molecules (the extracellular fluid (ECF)), other cells, and insoluble molecules that form the matrices of tissues. The molecular mix of the ECF, the behaviour of other cells, and the shape and chemical composition of the matrix change continuously. These changes are usually purposeful and vital for maintaining the integrity and function of the tissue and the cell must be able to respond to them. It does this by sampling the environment using complex molecular arrays on its surface that are part of the cell membrane. There are numerous families of surface molecules that respond to changes in the molecular composition of the $\mathrm{ECF}$, but the molecular mechanisms for responding to matrices and interacting directly with the surfaces of other cells are mediated largely by a single family of molecules called the cell adhesion molecules.

\section{Cell adhesion molecules}

CELL ADHESION MOLECULES ARE NOT JUST THERE TO PROMOTE ADHESION

These molecules are so named because at one level they allow cells to stick to other cells or cell matrices. But often this "stickiness" is not the prime purpose of the cell adhesion molecule. For the molecules on the surface of the cell to interact with molecules on the surface of adjacent cells and on neighbouring matrices the two molecules have to come into contact, a process that leads to the generation of intermolecular bonds that require a certain amount of energy to separate them. To break these bonds requires effort or energy and gives the impression of adhesion. If the purpose of the interaction between the two molecules had been to pass information from one molecule to the other, that purpose might have been disguised by the epiphenomenon of bond generation as part of the interactive processes of the two molecules. When viewed in this way it is easier to see that, although the result is cell adhesion, the primary function of the molecular interaction need not necessarily be just to moor or attach the cell to surrounding structures, but to allow the cell to respond to molecules attached to the surface of neighbouring cells or insoluble matrices. In many ways, the realisation that cell adhesion molecules were not simply cell attachment molecules, but response elements allowing the cell to react with its environment and so modify its biology to the advantage of the organism was a biological "eureka moment". Exactly how important that realisation was to understanding the biology of healthy and diseased cells is only now beginning to be realised completely. 
CELL ADHESION MOLECULES PAST AND PRESENT The story of cell adhesion molecules really comes from two separate and apparently unrelated areas of research. Much of the evidence that molecules responsible for cell interactions did actually exist came from studying leucocyte diapodesis from blood to tissue at sites of inflammation. The other impetus came from the study of embryology and, in particular, the migration of cells over and past one another during fetal development. However, only as the science has developed has it become apparent that the molecules involved in such diverse biological events are the same or closely related.

It is a feature of complex areas of molecular biology that during the phase of rapid growth and interest in an area of study there is a proliferation of concepts and names. One only has to look at the original names of the molecules that we now recognise by $C D$ numbers or the cytokines to realise how prevalent this trend has been. Such an examination also shows how important it is to have consensus meetings to pool knowledge and rationalise complex molecular groups. The cell adhesion molecules are equally good examples of this phenomenon. Although new cell adhesion molecules are being recognised every month, a rationalisation of these molecules has enabled them to be classified into six families on the basis of chemical, structural, or functional similarities.

THE FUNDAMENTAL FEATURES OF CELL ADHESION MOLECULES

Before describing these families it is important to understand that they all share three characteristics, indeed, the shared characteristics are more important than the differences.

(1) All cell adhesion molecules are glycoproteins and all act as a molecular link between the outside and the inside of the cell. Therefore, they are all transmembrane glycoproteins, spanning the cell membrane. As such, they have three different parts or domains (fig 1). The largest part is extracellular, the extracellular domain, and it is attached to the part within the membrane, the intramembranous domain, which in turn is attached to a part protruding into the cell, the cytoplasmic functional domain.

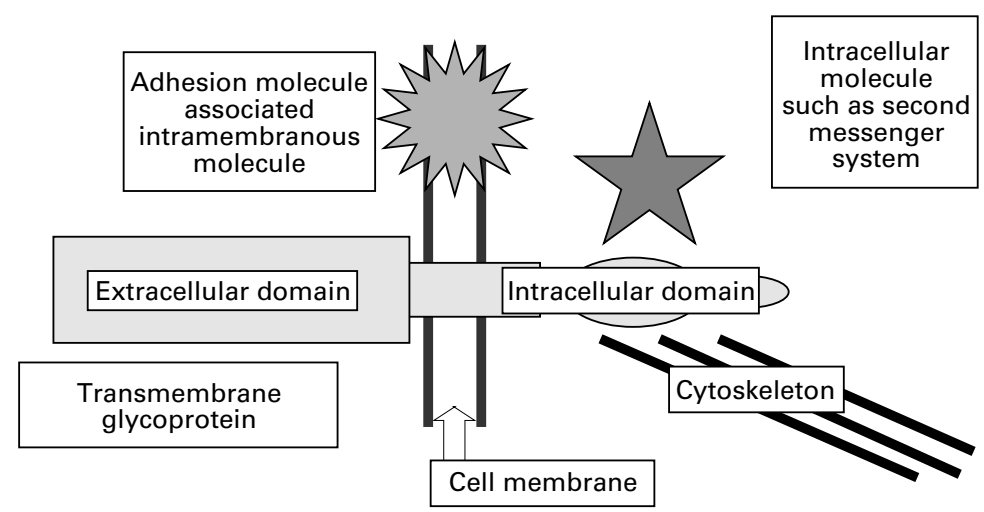

Figure 1 Structure and associations of a typical cell adhesion molecule.
(2) All the molecules work by an external stimulus (invariably another cell or matrix bound molecule) attaching to the extracellular domain and altering its structure. The molecule that binds to the cell adhesion molecule is very specific and is known as the cell adhesion molecule's receptor or ligand.

(3) The cell adhesion molecule is attached to another molecule within the cell through which it is able to influence the function of the cell. Some of these molecules are part of the cytoskeleton of the cell and others are enzymes or similar molecules that can induce a chemical change within the cell, usually operating through the so called "second messenger pathway".

THE FAMILIES OF CELL ADHESION MOLECULES

There are six families of cell adhesion molecules, they are:

(a) the immunoglobulin-like super family

(b) the cadherins

(c) the integrins

(d) the receptor protein tyrosine phosphatases

(e) the selectins

(f) the hyaluronate receptors.

There are some general properties of these molecules that help make them easier to understand, namely:

- Four of them $(\mathrm{a}-\mathrm{d})$ have ligands that are also proteins and two (e and $\mathrm{f}$ ) have ligands that are carbohydrates.

- All but the hyaluronate receptors are involved in cell-cell adhesion, integrins and hyaluronate receptors are involved in cellmatrix interactions.

- The ligands for the cell adhesion molecules fall into three groups:

(1) Molecules of the same group of cell adhesion molecules (homophilic). The cadherins fall into this category.

(2) Members of different groups of cell adhesion molecules. For instance the selectins are ligands for the immunoglobulin superfamily (and, of course, the opposite is also true).

(3) A molecule that is not a cell adhesion molecule. The integrins bind to $f$ matrix molecules through specific short peptide chains.

The immunoglobulin-like superfamily

This large family of molecules are so named because they have components that resemble immunoglobulins. ${ }^{1}$ The group includes molecules involved with cell and antigen recognition by lymphocytes and other cells. The family includes molecules used frequently by pathologists for cell recognition purposes including CD3, CD4, and CD8. Other important subgroups of these molecules are the intercellular adhesion molecules (ICAMs), which are expressed widely on epithelial and endothelial cells.

\section{Cadherins}

The calcium dependant cell adhesion molecules (cadherins) are so called because they have both adhesion and calcium binding sites. ${ }^{2}$ 
Typically, they bind to other cadherins on the surface of adjacent cells. Their cytoplasmic functional domains attach to a group of molecules known as catenins, which in turn attach to the intermediate filaments of the cytoskeleton. ${ }^{3}$ Effectively, the cadherin-catenin complex links the cytoskeleton to the changing environment outside the cell.

The best characterised cadherin is called $\mathrm{E}$ cadherin, a molecule whose expression is regulated by the ErbB2 proto-oncogene. ${ }^{4}$ Its expression is essential for early organisation of the developing embryo and, thus, it is one of the first adhesion molecules that humans express. E cadherin is concentrated in an intercellular junction known as the adherens junction, ${ }^{5}$ where it attaches to actin through the catenins. Cadherins are also important components of desmosomes. These cadherins are desmoglein and desmocollin ${ }^{6}$ which attach, again through catenins, to cytokeratins. ${ }^{7}$

The cadherins exemplify how our understanding of cell adhesion molecules is advancing. Recent studies of cadherins have shown them to be rather more diverse than was thought originally. Once believed to bind only to other cadherins and to be responsible for "epithelial" type binding, there is new evidence implicating them in lymphocyte-epithelial binding through cadherin-integrin bonds, and a new class of cadherins called type II cadherins that mediate "mesenchymal" loose adhesion have been described recently. One in particular, cadherin-11 (formally known as $\mathrm{OB}$ cadherin and first identified on osteoblasts ${ }^{8}$ ) is expressed widely at different stages of embryogenesis and might be essential for the organisation of mesenchyme derived tissues. ${ }^{9}$

Integrins

The integrins are both cell-cell and cell-matrix adhesion molecules. ${ }^{10}$ They do not consist of a single molecule but rather two different molecules lying side by side in the membrane. Molecules of this type are known as heterodimers. Integrin heterodimers consist of one $\alpha$ and one $\beta$ chain, both of which are necessary for ligand binding. Adhesion also requires the presence of divalent ions such as $\mathrm{Ca}^{2+}$ or $\mathrm{Mg}^{2+}$. To date, $14 \alpha$ and eight $\beta$ chains have been described. Theoretically, any combination of $\alpha$ and $\beta$ chain could exist but, so far, only limited permutations have been identified. Integrins are subclassified according to the $\beta$ subunit involved in the complex. ${ }^{11}$ Because many of the $\beta$ chains are rare, in humans there are only three main classes of integrins: $\beta 1, \beta 2$, and $\beta 3$. The $\beta 1$ and $\beta 3$ subfamilies are involved predominantly in interactions between cells and their matrices, while the members of the $\beta 2$ class are cell-cell adhesion molecules. There is an additional distinction between the $\beta 1$ and $\beta 3$ integrins in that, in general, the $\beta 1$ integrins are involved in adhesion to connective tissue macromolecules such as fibronectin, laminin, and collagens, whereas the $\beta 3$ integrins bind to vascular ligands such as fibrinogen, von Willebrand factor (factor VIII related antigen), thrombospondin, and vitronectin. In terms of cellular distribution, $\beta 1$ and $\beta 3$ integrins are coexpressed on most cell types, whereas $\beta 2$ integrins are restricted to leucocytes.

Some of the integrins are highly specific in their ligand binding properties, recognising short amino acid sequences on cell surfaces and matrix proteins; others are less specific. Thus, $\alpha 5 \beta 1$ binds to the tetrapeptide arginineglycine-aspartate-serine (RGDS) on fibronectin, whereas $\alpha 2 \beta 1$ binds to amino acid sequences on collagen, fibronectin, and laminin.

\section{Selectins}

Selectins have (lectin-like) carbohydrate binding domains on the extracellular component of the molecule. ${ }^{12}$ There are three main groups of selectins: the L selectins, which are homing receptors for specific adhesion of lymphocytes to endothelial cells of peripheral lymph nodes; the E selectins (endothelial leucocyte adhesion molecules), which are important mediators of inflammatory reactions; and $\mathrm{P}$ selectin, which is contained in Wiebel-Palade bodies of endothelial cells and $\alpha$ granules of platelets. This is released during clotting and at times of platelet activation, mediating adhesion between leucocytes and platelets.

\section{Hyaluronate receptors}

The fifth group is defined functionally, rather than structurally. Recently, this group has received increased interest because its members play a key role in determining growth, differentiation, and tumour progression. Hyaluronate is an abundant structural saccharide component of extracellular matrices that is believed to play an important role in a variety of physiological and pathological tissue processes, including inflammation, cell growth, cell migration, and tumorigenesis. Cells bind to hyaluronate through cell surface receptor proteins of which the best characterised is CD 44. ${ }^{13}$ CD44 displays molecular variation through a process known as alternative splicing, which influences the way in which mRNA is made from DNA. The various isoforms produced by alternative splicing can be nonhyaluronate binding, hyaluronate binding when activated, or constitutively binding. ${ }^{14}$ The CD44 isoforms have a variety of physiological roles including lymphocyte homing, immune response regulation (through lymphocyte activation), and cell migration.

\section{Receptor protein tyrosine phosphatases}

The receptor protein tyrosine phosphatases (RPTPs) are molecules involved in intercellular signalling and regulation of cell-cell adhesion. ${ }^{15} 16$ While the intercellular domains of other cell adhesion molecules need to link to enzymes or other molecules within the cytoplasm to influence intercellular events, the RPTPs have a functional cytoplasmic domain that resembles and works like the important intracellular molecule, tyrosine phosphatase. Through this component they have the potential to modulate intracellular events directly. 
Functions of cell adhesion molecules

Cell adhesion molecules allow cells to stick to one another and tissue matrices. They also enable a cell to recognise and react to events within its local environment that are mediated directly by these cells and matrices. They do this by having a functional component either side of the cell membrane. Therefore, by responding to events outside the cell they are able to induce profound changes in cell shape, cell function, and nuclear gene transcription, either through their attachments to the structural units of the cell (the cytoskeleton) or through enzymes that initiate intracellular chemical cascades. These abilities place these molecules in a privileged position to drive changes in cell function.

The types of functional change that these molecules can initiate are fundamental to the way in which organisms work and develop, and these molecules are important targets for the initiation and propagation of pathological events.

\section{INTRACELLULAR AND EXTRACELLULAR EVENTS} THAT CAN BE MEDIATED BY CELL ADHESION MOLECULES

Some of the functions of cell adhesion molecules have already been alluded to. It is clear that, at a cellular level, cell adhesion molecules are responsible for more than just adhesion of cells to one another and to their insoluble matrices. Additional functions include the ordering of cell sorting, migration and differentiation; organisation of cell motility via the cytoskeleton; regulation of intercellular and intracellular signalling; and control of gene transcription

\section{Cell-cell adhesion}

Cell-cell adhesion is an important function of cell adhesion molecules. Adhesion of one cell to another serves two major purposes: (1) to form and allow coordinated function of distinct groups, lines, or sheets of cells; and (2) to prevent or arrest cell movement. This is achieved both through specialised cell junctions such as desmosomes, in which cadherins are important structural and functional components, and through single or closely clustered individual molecules. The pathologist's daily observation that in certain disorders, such as inflammation,

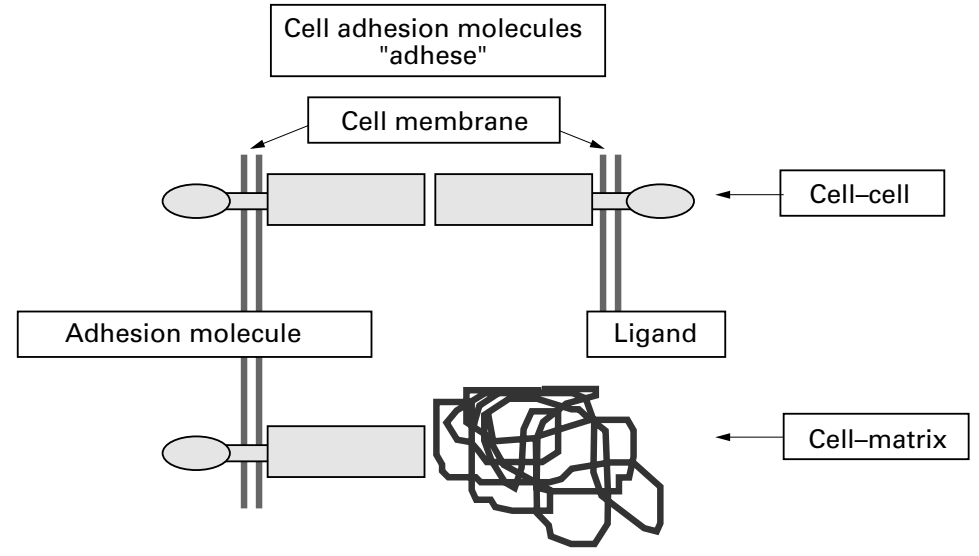

Figure 2 Adhesion molecules in cell-cell adhesion and cell-matrix adhesion. cell-cell adhesion is upregulated, while in others, such as tumour infiltration, loss of adhesion is occurring, has made this effect of cell adhesion molecules a particular area of interest among pathologists (fig 2).

\section{Cell-matrix adhesion}

Integrins are the predominant mediators of cell-matrix adhesion. It is now clear that these molecules are a key part of a very complex organisational hierarchy that controls normal cell function. For example, osteoblasts form a monolayer with every cell adherent to its matrix, and endothelial cells and many epithelial cells are tightly organised to perform specific functions by their underlying matrix. Cell-matrix interactions are also key to understanding pathological processes-for example, one of the important ways by which early malignancy is recognised is by cells breaking free of the constraints usually imposed upon them by their basement membranes.

\section{Regulation of cell signalling}

In addition to their role in cell adhesion, cell adhesion molecules are involved in the transmission of signals across cell membranes. It is now known that these signals can be of two types. The first and more obvious is signal passage from the outside of the cell to the inside in response to ligand binding, the activation of second messenger systems, and gene transcription. Such signals are responsible for functions as diverse as stimulation of anchorage dependant cell growth and differentiation and protection from apoptosis. ${ }^{17}{ }^{18}$ The second is passage of information from the inside of the cell to the outside ("inside out" signalling). This usually modulates the binding affinities of cell adhesion molecules. ${ }^{19}$ It is implicit that for cell adhesion molecules to be involved in both mechanisms, certain intercellular and intracellular signalling pathways must be focused upon cell adhesion molecules or other molecules concentrated in their immediate locality.

One of the most remarkable facets of cell biology is that many signals passing into the cell from outside are transmitted within the cell by second messenger systems. There is no evidence to suggest that the intracellular signalling pathways activated by binding of cell adhesion molecules to their ligands are in any way specific - they are the same second messenger systems that are activated by many other receptor-ligand binding systems, ${ }^{20}$ such as cytokines-cytokine receptors.

\section{Cell motility via the cytoskeleton}

Cell adhesion molecules are thought to play an important role in controlling cell migration through their connections with the cytoskeleton (fig 3). For instance, through their cytoplasmic domains, cadherins associate with catenins, which in turn are attached to contractile molecules such as actin. ${ }^{3}$ It is also known that in certain disorders where cell adhesion molecules are abnormal, such as leucocyte adhesion deficiency, cell motility is also abnormal. 
Many adhesion (such as cadherin) molecules are attached via their cytoplasmic domains to cytoskeletal components through catenin molecules to actin

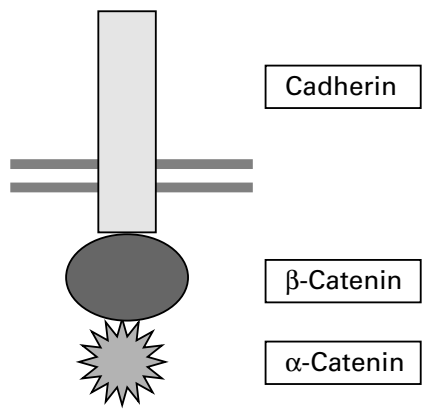

Actin

Figure 3 Adhesion molecules control cell motility through their connections with the cytoskeleton.

For directed movement there is evidence of migration of cell adhesion molecules to specific areas of the membrane
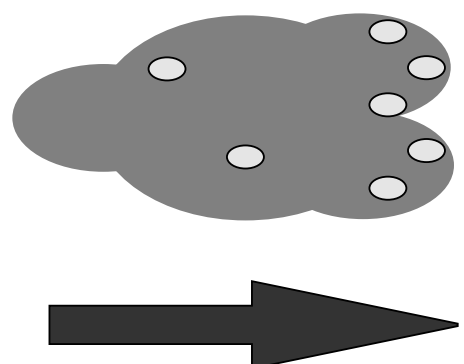

Figure 4 Adhesion molecules are involved in directed cell motility.
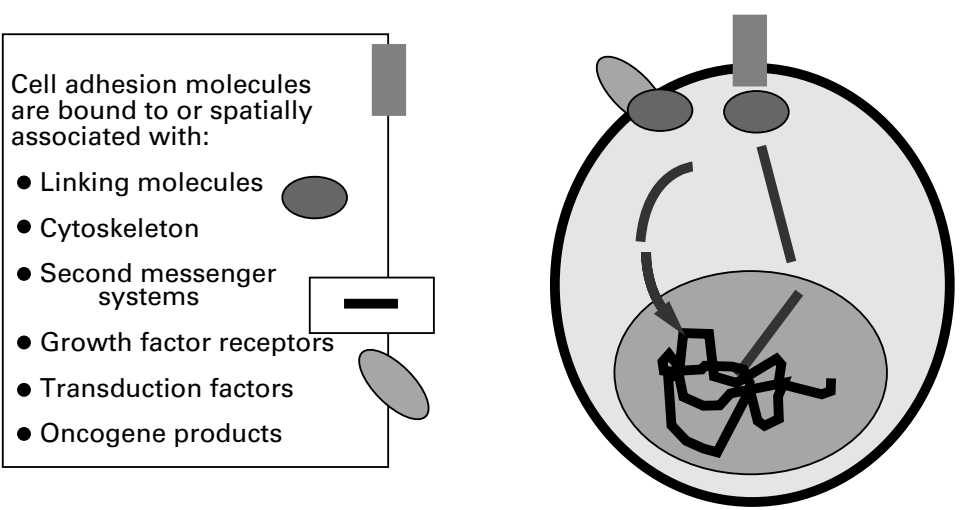

Figure 5 Adhesion molecules regulate gene transcription.

Despite observations such as this, the roles of cell adhesion molecules and their receptors in the control of cell movement via connections with the cytoskeleton are still poorly understood. However, it is believed that cell motility across a matrix is probably regulated through a cycle of attachment and detachment mediated through cell adhesion molecules. For this to occur, cells would have to extend a cytoplasmic process that becomes tightly adherent at its tip. The cell would then pull itself towards the attachment point, finally detaching from this point after having sent out another process and forming a new attachment point. ${ }^{21}$ To achieve this efficiently, cells would need to cause migration and aggregation of cell adhesion molecules within their cell membranes, and there is evidence that this does indeed occur ${ }^{22}$ (fig 4).

Regulation of gene transcription

Cell adhesion molecules can influence both the cytoskeleton and common second messenger systems via their intracytoplasmic domains. There is evidence (direct and indirect) that cell adhesion molecules can influence gene transcription through these mechanisms (fig 5). This is best exemplified by examining the effects of alterations in the matrix on gene expression of cultured cells. In such experiments, the general culture conditions are kept uniform and the only factors that change are the shape and/or structure of the matrix. ${ }^{23}$ Experiments on hepatocytes-for example, have shown that if they are maintained on a malleable matrix, the expression of early response genes (such as myc) is downregulated, differentiation of the cell occurs, and the DNA binding activity of transcription factors such as AP-1 is attenuated. ${ }^{24}$ There is also evidence from other studies that the expression of genes in the liver is upregulated in a manner that is dependant upon cell shape. ${ }^{25}$

Whether these effects on gene transcription are mediated via second messenger systems or the cytoskeleton has yet to be elucidated fully. It is of interest to note, in this context, that the nuclear skeleton has attachments to the cytoskeleton $^{26}$ through nuclear laminins ${ }^{27}$ and that the nuclear skeleton incorporates a series of protein fibres that bind to DNA at "matrix attachment regions". It is possible that cell adhesion molecules can cause changes in the cytoskeleton that in turn restructure the nuclear matrix, initiate changes in DNA binding, and so influence gene expression.

\section{Cell migration, sorting, and differentiation}

Nowhere is the interplay between cell migration, sorting, and differentiation better illustrated than in the area of embryogenesis and morphoregulation. Indeed, one of the major factors initiating the intense examination of cell adhesion molecules over recent years has been the study of cell sorting phenomena proposed to orchestrate morphogenesis. The organisation of multicellular organisms requires the selective association of embryonic cells into specific tissues. The pattern of expression of cell adhesion molecules, notably the members of the immunoglobulin-like and cadherin families, indicates that these molecules play a pivotal role in linking the primary processes of cell division, migration, differentiation, and cell death, so that their temperospatial expression make them prime candidates for morphoregulation $^{28}$ (fig 6). More is known, albeit often indirectly, about the role of the different families of cell adhesion molecules in this context than any other.

\section{Specific examples of cell adhesion molecules and their ligands in normal and diseased tissue}

To give an idea of how important these molecules are to the regulation of body, tissue, and cell function, the next few paragraphs illustrate a tiny amount of what is known about their role in healthy and diseased tissues. 


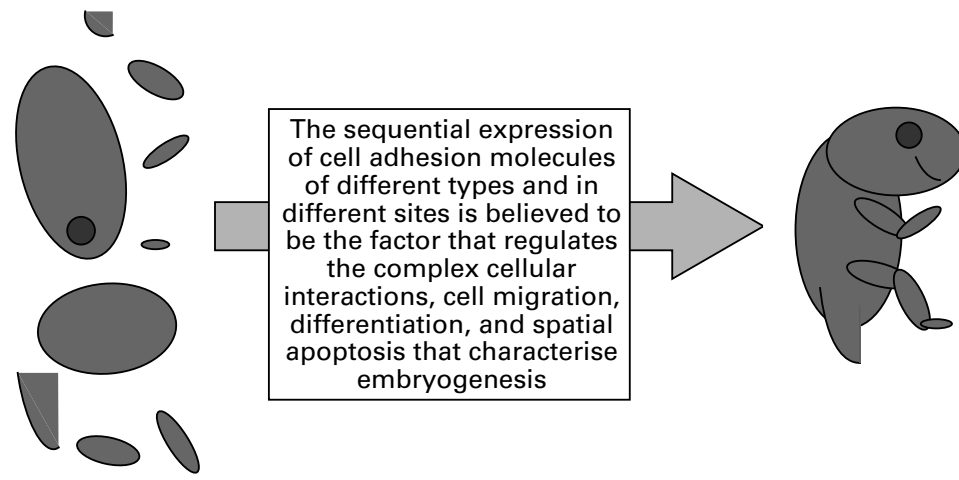

Figure 6 Adhesion molecules play an important role in cell migration, sorting, and differentiation during embryogenesis.

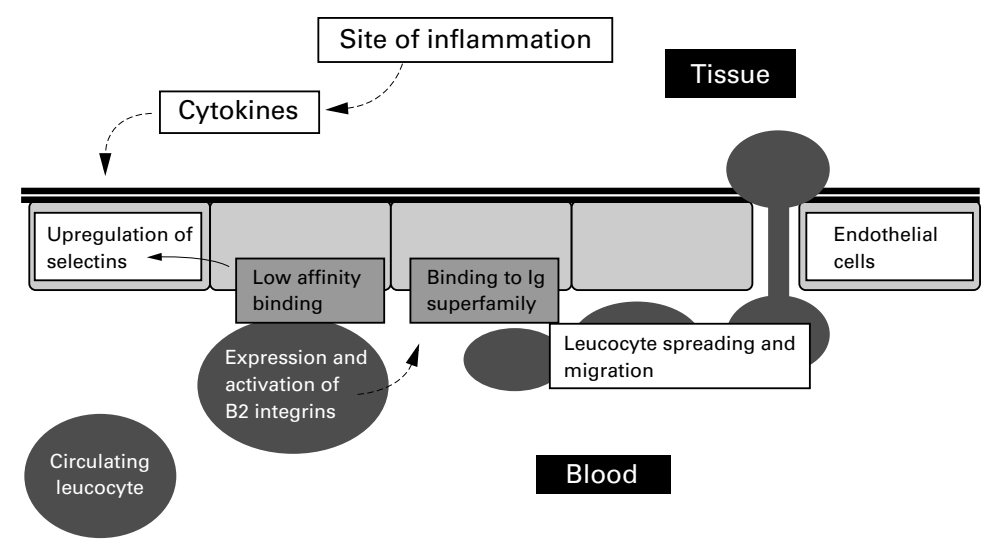

Figure 7 Adhesion molecules in leucocyte trafficking.

CELL ADHESION MOLECULE FUNCTION IN NORMAL TISSUES

Embryogenesis

One of the major factors initiating the early examination of cell adhesion molecules was the study of cell sorting phenomena occurring during morphogenesis. The organisation of multicellular organisms requires the selective association of embryonic cells into specific tissues. Cell recognition and sorting is mediated by the different classes of cell adhesion molecules that are present on the surface of embryonic cells. ${ }^{28}$ Examples of these are: (a) the cadherins that are known to regulate a variety of early events in embryogenesis, some of which are very complex, such as the interactions between ectoderm and mesoderm that lead to the formation of the neural plate ${ }^{29}$; (b) CD44 in the development of the limb bud where, in animals, antibodies to CD44 epitopes block cartilage formation in cultured limb bud cells. ${ }^{30}$

\section{Myogenesis and muscle function}

It is now well recognised that muscles rely for their number and position, and particularly their complex relationships with the nervous system, on the controlled expression of cell adhesion molecules. Most important among these are the cadherins. For instance, $T$ cadherin is absent from muscle cells during early development and is upregulated once innervation has occurred and synapses formed. Furthermore, unlike $\mathrm{N}$ cadherin, which appears to anchor the nerve to the synaptic site, $T$ cadherin is not present at synapses, leading to the inference that $\mathrm{T}$ cadherin inhibits the sprouting of accessory nerve terminals. ${ }^{31}$

Neural function

Cadherins have also been implicated in nerve growth, ${ }^{32}$ such that $\mathrm{N}$ cadherin, $\mathrm{E}$ cadherin, and $T$ cadherin delineate different regions in the developing spinal cord. The complementary distribution of these molecules is particularly evident in dorsal root ganglia.

CELL ADHESION MOLECULES IN PATHOLOGICAL PROCESSES

Adhesion molecules may, through normal or abnormal expression, modulate disease processes. ${ }^{33}$ The role of cell adhesion molecules (or their ligands) in pathological processes can be thought of as occurring in two situations: (1) as part of a physiological response to pathological situations (such as inflammation); (2) as part of the pathological process itself.

Exploitation of cell adhesion molecules by infective organisms

Viruses-Viruses and other organisms can exploit normal cell adhesion molecules for tissue binding. For example, the human immunodeficiency virus (HIV) binds to T lymphocytes through the CD4 adhesion molecule, whereas other viruses express the RGDS motif on their surfaces, enabling them to bind to target cell surfaces via integrins before entry into the cell. ${ }^{34} 35$

Protozoa-Intercellular adhesion molecules are also implicated in the adhesion of red blood cells infected with malarial organisms to capillary endothelial cells. Cytokine mediated upregulation of endothelial intercellular adhesion molecules might be associated with the severity of this disease. ${ }^{36}$

Bacteria-The Lyme disease spirochete, Borrelia bergdorfi, binds to platelets through $\alpha 11 \beta 3$ in an interaction inhibited by the RGDS peptide. Binding of the organism to epitopes expressed on platelets might be of importance in its dissemination. ${ }^{37}$ Similarly, cadherins are exploited by bacteria such as the gram negative enteric bacillus Shigella flexneri, which binds to them before invading colonic epithelial cells, probably using $\mathrm{N}$ cadherin. ${ }^{38}$

\section{Inflammation}

One aspect of inflammation that has been studied intensively from the perspective of cell adhesion molecules is leucocyte cell trafficking from blood to tissue (fig 7). Endothelium becomes more adhesive for circulating "inflammatory" cells by altered, often sequential, expression of different cell adhesion molecules. ${ }^{39}$ For instance, early in the inflammatory response, there is increased expression of selectins on endothelial cells stimulated by inflammatory mediators such as cytokines. The selectins mediate a low affinity interaction with leucocytes that serves to concentrate them on endothelial surfaces at the site of injury. The transient presence of leucocytes on the endothelium stimulates expression and activation of $\beta 2$ integrins on the leucocytes. These, in 
turn, interact with members of the immunoglobulin superfamily expressed by endothelial cells, resulting in stronger adhesion and leucocyte spreading.

A number of concepts of value in the general understanding of cell adhesion molecule biology emanate from these observations, including: (a) although families of cell adhesion molecules can have diverse actions, molecules from different families might have similar functions; (b) soluble informational molecules such as cytokines can regulate cell adhesion molecule expression; (c) the activity of one cell adhesion molecule can lead to changes in the expression of another.

It is likely that these concepts are not restricted to inflammation but are found more generally in other complex phenomena, such as the development and dissemination of neoplasms and embryonic development.

\section{Wound healing and angiogenesis}

Studies focusing on integrin expression during wound re-epithelialisation have shown a temporal sequence of parallel physical and chemical changes in keratinocytes during the repair process. Keratinocytes at the wound margin begin migrating over the wound very soon after injury. Compared to stationary basal keratinocytes, migrating keratinocytes express large amounts of $\alpha 5 \beta 1$ and $\alpha \mathrm{V} \beta 5^{40}$ (fig $8)$. The induction of this set of integrins might be necessary for keratinocyte migration because they engage ligands such as fibronectin or vitronectin that are found in the blood clot matrix. ${ }^{41}$ Cytokine stimulation and altered keratinocyte matrix are probably among the conditions that disrupt the normal expression and distribution of integrins during re-epithelialisation. ${ }^{42}{ }^{43}$

Integrins also contribute to the growth of new blood vessels during wound repair. The integrin $\alpha \mathrm{V} \beta 3$ is expressed on blood vessels in granulation tissue, but not normal skin, and antibodies against $\alpha \mathrm{V} \beta 3$ block angiogenesis in vivo. ${ }^{44}$ Furthermore, endothelial cell $\alpha \mathrm{V} \beta 3$, which binds to fibrinogen, appears to regulate the formation of capillary tubes in fibrin gels, while $\alpha 2 \beta 1$, which binds to collagen, similarly regulates tube formation in collagen gels. ${ }^{45}$

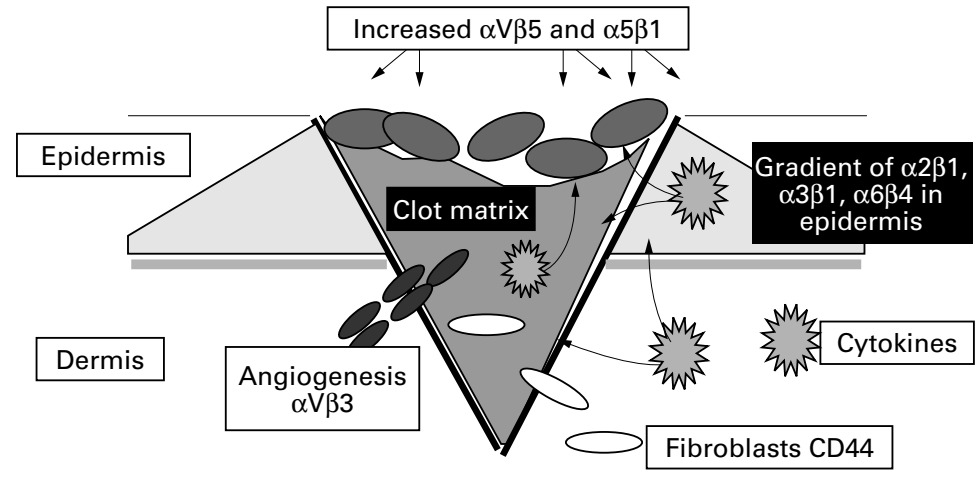

Figure 8 Adhesion molecules play an important role in wound healing.
PATHOLOGICAL PROCESSES MEDIATED BY ABNORMALITIES IN CELL ADHESION MOLECULES Immunodeficiency disorders

The inherited immunodeficiency disease leucocyte adhesion deficiency, caused by defects in the common $\beta 2$ subunit of leucocyte integrins, leads to the paradox of granulocytosis with lack of pus formation at sites of bacterial infection. The defect in the $\beta 2$ molecule might occur either by mutation or abnormal translation of mRNA. ${ }^{46}$ Patients with this disease suffer from recurrent or persistent infections.

\section{Bleeding disorders}

Mutation of the $\beta 3$ subunit of the platelet integrin platelet glycoprotein IIb/IIIa is the cause of Glanzmann's throbasthenia. ${ }^{47}$

\section{Blistering skin diseases}

In pemphigus, blistering is caused by antibodies to desmosomal constituents, particularly the cadherins. ${ }^{48}$ Complement induced, antibody mediated damage causes the desmosomes to break down, with separation of keratinocytes and intra-epidermal blister formation.

In bullous pemphigoid, antibodies against components of the keratinocytes' basement membrane binding hemidesmosomes are present in the serum. The hemidesmosome adhesion receptor is $\alpha 6 \beta 4$ integrin. Damage to the hemidesmosome leads to failure of adhesion between the epidermis and its basement membrane, with the formation of subepidermal blisters.

\section{Neoplasia}

Malignancy is characterised by invasion and metastasis. Theoretically, invasion into surrounding tissue and metastasis should require profound and contradictory changes in cell adhesiveness. This has been found to be the case, but it is also becoming clear that adhesive phenomena in tumour dissemination and metastasis, while possessing some unique features, also have parallels with the inflammatory response. The latter is not surprising because metastatic cells enter and exit the circulatory systems in similar ways to inflammatory cells.

A fairly good correlation exists between the loss of $\mathrm{E}$ cadherin expression and the acquisition of the undifferentiated state. Particularly striking is the case of diffuse-type gastric carcinoma. ${ }^{49}$ Over $50 \%$ of tumours studied showed deletions in $\mathrm{E}$ cadherin exon 8 or 9 .

In a series of different human tumour cell lines, invasion into collagen gels was inversely related to expression of $\mathrm{E}$ cadherin and inhibition of invasion was achieved by transfection with a vector expressing $E$ cadherin, suggesting a metastatic suppresser function for this cell adhesion molecule. ${ }^{50}$

In an extensive study of genetic aberrations in colonic carcinomas a common deletion was identified in a gene (DCC) that codes for a protein with homology to the immunoglobulinlike superfamily of adhesion molecules. ${ }^{51}$ 
A multitude of different changes in integrin expression have been reported in tumours. Adhesion to endothelial cells is presumed to play an important roll in the act of haemic and lymphatic metastasis. There is evidence from experimental tumour systems that this is mediated by $\beta 1$ and $\beta 3$ integrins, and can be inhibited by short synthetic peptides that bind to the RGDS recognition site. ${ }^{52}$

Selectins might also play an important role in metastasis. Potential endothelial ligands for $\mathrm{L}$ selectin can be seen on venules in a high proportion of cutaneous lymphomas, suggesting that they might be involved in the spread of these lymphomas. ${ }^{53}$ There is also evidence that the expression of carbohydrate determinants for $\mathrm{E}$ selectin binding is related to the metastatic potential of colon carcinoma cells. ${ }^{54}$ By a similar mechanism, P selectin on endothelial cells could also be involved in the metastatic arrest of tumour cells bearing appropriate carbohydrate epitopes.

CD44 isoforms have been detected on a variety of metastasising experimental tumour cell lines, but not on normal cells of the same lineage or non-metastasising variants of the same tumour. ${ }^{55}$ After injection into the skin, CD44 positive rat carcinoma cells formed lymph node metastases, followed by lung infiltrates. The role of CD44 in the process of metastasis in this model has been demonstrated in two ways. First, if antibodies against certain CD44 epitopes were injected at the same time as the tumour cells, metastatic spread was delayed. ${ }^{56}$ Secondly, transfection of non-metastasising rat carcinoma cells with certain isoforms of CD44 rendered the tumour metastasising.

The specific isoforms implicated in metastatic potential are the group called CD44v. Several human tumours have been investigated using immunohistochemistry and the polymerase chain reaction for evidence of CD44v expression. In colonic and breast cancers, a direct relation has been described between isoform type and metastatic potential. In the most aggressive tumours, up to $100 \%$ of cases expressed CD44v, whereas the equivalent normal epithelial cells did not express this molecule. ${ }^{57}$

\section{Tumour suppresser genes}

Recently, it has become evident that the proper formation of intercellular junctions is critical for the maintenance of epithelial differentiation and that destabilisation of junctions allows epithelial cells to invade and carcinomas to progress. ${ }^{58}$ Accordingly, various structural components of intercellular junctions have been found to be related to products of tumour suppresser genes. The evidence for a tumour suppresser function for $\mathrm{E}$ cadherin has been outlined above. The tumour suppressor gene product APC binds to $\beta$-catenin, which is also cytoplasmically associated with $\mathrm{E}$ cadherin, ${ }^{59}$ and the neurofibromatosis 2 tumour suppresser gene product merlin is a member of the moesin-ezrin-radixin family of junctional proteins. ${ }^{60}$ Conversely, products of oncogenes such as src, ras, fos, and met have been shown to destabilise intercellular junctions ${ }^{61}$; for src (the gene encoding the epidermal growth factor receptor) and met this occurs through phosphorylation of $\beta$-catenin at tyrosine residues. ${ }^{62}$

\section{The significance of cell adhesion molecules to the diagnostic histopathologist}

It is interesting to speculate on how the wealth of knowledge accumulated through the application of molecular pathology techniques to the study of cell adhesion molecules can be exploited by the diagnostic histopathologist. After all, these molecules are responsible for, or at least involved in, everything that happens to cells from embryogenesis to apoptosis, from cell motility to cell adhesion, and from immune regulation to tumour metastasis. Cell adhesion molecules, therefore, have an intellectual significance to those who, like pathologists, have an interest in disease.

The rate of discovery of new adhesion molecules has declined sharply in recent years, and it now seems that most of the main adhesion molecules and their alternatively spliced forms have been found. A large amount of literature has grown up around these molecules, but there is still a need to define their nature and significance in human disease. Cell adhesion molecules are pivotal in tissue assembly, both during development and in the adult, and are essential for understanding the mechanisms of inflammation, wound healing, vascular integrity and growth, and neoplasia. There is considerable scope for the histopathologist to be involved in, and direct research into, the nature and effects of differential expression of cell adhesion molecules in diseased tissues. Recognition of these mechanisms also offers attractive targets for novel treatments.

Intellectual curiosity and applied research aside, it is relevant to ask whether current data can be used. Many data look encouraging in the sense that they could be used to diagnose and to prognosticate. There are two particular areas that stand out as being important ones for the tissue pathologist. The first is in cell recognition and the second is the broad field of neoplasia.

Some of the cell adhesion molecules are relatively cell specific and can, and are, being used to identify cell types. Perhaps the most commonly used, currently, are the immunoglobulin super family molecules such as CD3. Others are also cell specific: L selectin will identify high endothelial venules and cadherin-11 will identify osteoblasts. The last is of more than passing interest because it illustrates one way in which the cell adhesion molecules might be exploited in diagnosis. Alternatively spliced variants of $\mathrm{OB}$ cadherin exist but are only found in neoplastic osteoblasts. ${ }^{63}$ Therefore, identifying the spliced variants could form the basis of recognising neoplastic, as opposed to reactive, bone.

Arguably, it is in the area of neoplasia that the study of cell adhesion molecules offers the greatest gains for the histopathologist. Diagnostic tests, similar to the one outlined above 


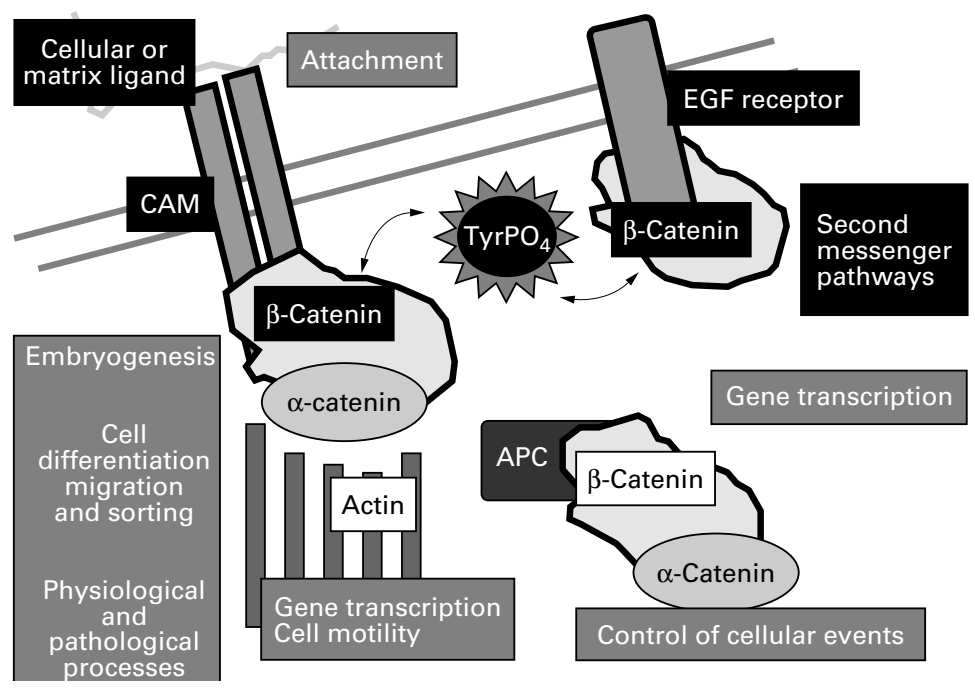

Figure 9 Summary of events mediated by cell adhesion molecules.

for osteoblastic neoplasms, based on abnormal expression of molecules, usually associated with stable intercellular or cell-matrix junctions, will be developed. Already there are reports of increased concentrations of soluble $\mathrm{E}$ cadherin $^{64}$ and $\mathrm{CD} 44^{65}$ in the circulation of patients with certain cancers, and the detection of unusual spliced variants of CD44 in exfoliated cells in urine has been shown to have some correlation with the presence of transitional cell neoplasia. ${ }^{66}$ Perhaps even more important to the histopathologist will be tests with prognostic significance. The loss of $\mathrm{E}$ cadherin expression, while clearly distinguishing benign cells from malignant ones, is said to reflect more accurately their differentiation, metastatic and invasive potential, and prognosis in a variety of carcinomas including those of the stomach, endometrium, ${ }^{67}$ prostate, ${ }^{68}$ and breast. ${ }^{69}{ }^{70}$ Much the same applies to CD44 variant expression in lung, ${ }^{71}$ cervix, ${ }^{72}$ breast, ${ }^{73}$ and colorectal carcinomas. ${ }^{74}$

A particularly good example of how this type of information might be applied comes from two studies of colonic carcinoma. In the first, the differential ability of human colonic carcinoma cell lines to bind to $\mathrm{E}$ selectin reflected directly their potential to metastasise to the liver. ${ }^{75}$ In the second, there was an excellent positive correlation between expression of the $\mathrm{E}$ selectin ligand, sialyl Lewis $\mathrm{X}$ antigen, on human colorectal carcinoma cells and the incidence of distant metastasis and five year survival rate. ${ }^{54}$

\section{Summary}

The cell adhesion molecules are ubiquitous recognition molecules that allow cells to communicate with one another and their environment. Through these molecules, complex alterations in the cytoplasmic messenger pathways and the microfilamentous cytoskeleton can lead to profound alterations in cell division, differentiation, behaviour, and function (fig 9). It is difficult to conceive of a group of molecules that could be more important to pathologists and to their understanding of disease processes.
1 Springer TA. Adhesion receptors of the immune system. Nature 1990;346:425-34.

2 Takeichi M. Cadherins: a molecular family important in selective cell-cell adhesion. Annu Rev Biochem 1990;59: 237-52.

3 Garrod DR. Desmosomes and hemidesmosomes. Curr Opin Cell Biol 1993;5:30-40.

4 D'Souza B, Taylor-Papadimitriou J. Overexpression of ERBB2 in human mammary epithelial cells signals inhibition of transcription of the E-cadherin gene. Proc Natl Acad Sci USA 1994;91:7202-6.

5 Nathke IS, Hinck L, Swedlow JR, et al. Defining interactions and distributions of cadherin and catenin complexes in polarised epithelial cells. $\mathcal{F}$ Cell Biol 1994;125:1341-52.

6 Buxton RS, Cowin P, Franke WW, et al. Nomenclature of the desmosomal cadherins. F Cell Biol 1993;121:481-3.

7 Schafer S, Troyanovsky S, Heid HW, et al. Cytoskeletal architecture and epithelial differentiation: molecular determinants of cell interaction and cytoskeletal filament anchorage. C R Acad Sci III 1993;316:1324-31.

8 Okazaki M, Takeshita S, Kawai S, et al. Molecular cloning and characterisation of OB-cadherin, a new member of cadherin family expressed in osteoblasts. F Biol Chem 1994; 269:12092-8.

9 Kimura Y, Matsunami H, Inoue $\mathrm{T}$, et al. Cadherin-11 expressed in association with mesenchymal morphogenesis in the head, somite, and limb bud of early mouse embryos. Biol Dev 1995;169:347-58.

10 Hynes RO. Integrins: a family of cell surface receptors. Cell 1987;48:549-50.

11 Hynes RO. Integrins: versatility, modulation and signalling in cell adhesion. Cell 1992;69:11-25.

12 Lasky LA. Selectins: interpreters of cell-specific carbohydrate information during inflammation. Science 1992;258: 964-9.

13 Peach RJ, Hollenbaugh D, Stamenkovic I, et al. Identification of hyaluronic acid binding sites in the extracellular domain of CD 44. F Cell Biol 1993;122:257-64.

14 Dougherty GJ, Cooper DL, Memory JF, et al. Ligand binding specificity of alternatively spliced CD44 isoforms. Recognition and binding of hyaluronan by CD44R1. F Biol Chem 1994;269:9074-8.

15 Tonks NK (ed). Protein tyrosine phosphates. Semin Cell Biol 1993;4:373-453.

16 Beckman G, Bork P. An adhesive domain detected in functionally diverse receptors. Trends Biochem Sci 1993;18: $40-1$.

17 Guadagno TM, Ohtsubo M, Roberts JM, et al. A link between cyclin A expression and adhesion-dependant cell cycle progression. Science 1993;262:1572-5.

18 Meredith J, Fazeli B, Schwartz MA. The extracellular matrix as a cell survival factor. Mol Biol Cell 1993;4:95361.

19 Humphries MJ, Mould AP, Tuckwell DS. Dynamic aspects of adhesion receptor function: integrins both twist and shout. BioEssays 1993;15:391-7.

20 Schwartz MA, Ingber DE. Integrating with integrins. Mol Biol Cell 1994;5:389-93.

21 Lee J, Ishihara A, Jacobson K. How do cells move along surfaces. Trends Cell Biol 1993;3:336-70.

22 Kusumi A, Sako Y, Yamamoto M. Confined lateral diffusion of membrane receptors as studied by single particle tracking (nanovid microscopy). Effects of calcium-induced differentiation in cultured epithelial cells. Biophys $\mathcal{f}$ 1993;65:2021-40.

23 Hynes RO. Genetic analyses of cell-matrix interactions in development. Curr Opin Genet Dev 1994;4:569-74.

24 Rana B, Mischoulon D, Xie Y, et al. Cell-extracellular matrix interactions can regulate the switch between growth and differentiation in rat hepatocytes: reciprocal expression and differentiation in rat hepatocytes: reciprocal expression
of $\mathrm{C} / \mathrm{EBP}$ and immediate-early growth response transcripof C/EBP and immediate-early growth respo
tion factors. Mol Cell Biol 1994;14:5858-69.

25 DiPersio CM, Jackson DA, Zaret KS. The extracellular matrix co-ordinately modulates liver transcription factors and hepatocyte morphology. Mol Cell Biol 1991;11:440514

26 Pienta KJ, Coffey DS. Nuclear-cytoskeletal interactions: evidence for physical connections between the nucleus and cell periphery and their alteration by transformation. $f$ Cell Biochem 1992;49:357-65.

27 Georgatos SD. Towards an understanding of nuclear morphogenesis. F Cell Biochem 1994;55:69-76.

28 Hynes RO, Lander AD. Contact and adhesive specificities in the associations, migrations, and targeting of cells and the associations, migrations,
axons. Cell 1992;68:303-22.

29 Haesman J, Ginsberg D, Geiger B, et al. A functional test for maternally inherited cadherin in Xenopus embryos shows its importance in cell adhesion at the blastula stage. its importance in cell adhe

30 Toole BP, Turner RE, Banerjee SD. Hyaluronan-binding protein in chondrogenesis and angiogenesis in the developing limb. Prog Clin Biol Res 1993;383B:437-44

31 Fredette B, Rutishauser U, Landmesser L. Regulation and activity-dependence of N-cadherin, NCAM isoforms and polysialic acid on chick myotubes during development. f Cell Biol 1993;123:1867-88.

32 Redies C, Engelhardt K, Takeichi M. Differential expression of $\mathrm{N}$ - and R-cadherin ion functional neuronal systems and other structures of the developing chicken brain. 7 Comp other structures of the develo
Neurol 1993;333:398-416.

33 Humphries MJ. The molecular basis and specificity of integrin-ligand interactions. F Cell Sci 1990;97:585-92. 
34 Logan D, Abu-Ghazaleh R, Blakemore W, et al. Structure of a major immunogenic site on foot-and-mouth disease virus. Nature 1993;362:566-8.

35 Wickham TJ, Matthias P, Cheresh DA, et al. Integrins $\alpha \mathrm{V} \beta 3$ and $\alpha \mathrm{V} \beta 5$ promote adenovirus internalisation but not virus attachment. Cell 1993;73:309-19.

36 Berendt AR, Simmons DC, Tansey J, et al. Intercellular adhesion molecule-1 in an endothelial cell adhesion receptor for Plasmodium falciparum. Nature 1989;341:57-9.

37 Coburn J, Leong JM, Urban JK. Integrin $\alpha 11 \beta 3$ mediates binding of the Lyme disease agent Borrelia burgdorferi to human platelets. Proc Natl Acad Sci USA 1993;90:705963.

38 Sansonetti PJ, Mounier J, Prevost MC, et al. Cadherin expression is required for the spread of Shigella flexneri between epithelial cells. Cell 1994;76:829-39.

39 Springer TA. Traffic signals for lymphocyte recirculation and leukocyte emigration: the multistep paradigm. Cell 1994;76:301-14.

40 Kurpakas MA, Quaranta V, Jones JCR. Surface relocation of $\alpha 6 \beta 4$ integrins and assembly of hemidesmosomes in an in
vitro model of wound healing. F Cell Biol 1991;115:1737vitro

41 Cavani A, Zambruno G, Marconni A, et al. Distinctive integrin expression in the newly forming epidermis during wound healing in humans. F Invest Dermatol 1993;101:6004.

42 Gailit J, Welch MP, Clark RAF. TGF- $\beta 1$ stimulates expression of keratinocyte integrins during re-epithelialization of cutaneous wounds. F Invest Dermatol 1994;103:221-7.

43 Adams JC, Watt FM. Regulation of development and differentiation by the extracellular matrix. Development 1993 117:1183-98.

44 Brooks PC, Clark RAF, Cheresh DA. Requirement of vascular

45 Gamble JR, Matthias LJ, Mayer G, et al. Regulation of in vitro capillary tube formation by anti-integrin antibodies. $\mathcal{F}$ Vitro capillary tube formation

46 Hibbs ML, Wardlaw AJ, Stacker SA, et al. Transfection of cells from patient with leukocyte adhesion deficiency with cells from patient with leukocyte adhesion deficiency with
an integrin $\beta$ subunit (CD18) restores lymphocyte an integrin $\beta$ subunit (CD18) restores lymphocyte function-associated antigen-1.

47 Gawaz MP, Loftus JC, Bajt ML, et al. Ligand bridging mediates integrin $\alpha 11 \beta 3$ (platelet GPIIB-IIIA) dependan homotypic and heterotypic cell-cell interactions. F Clin Invest 1991;88:1128-34

48 Stanley JR. Cell adhesion molecules as targets of autoantibodies in pemphigus and pemphigoid, bullous diseases due to defective epidermal cell adhesion. Adv Immunol 1993;53:291-325.

49 Becker KF, Atkinson MJ, Reich U, et al. E-cadherin gene mutations provide clues to diffuse type gastric carcinomas. Cancer Res 1994;54:3845-52.

50 Vleminckx K, Vakaet L, Mareel M Jr, et al. Genetic manipulation of E-cadherin expression by epithelial tumour cells

51 Fearon ER, Vogelstein B. A genetic model for colorectal tumorigenesis. Cell 1990;61:759-67.

52 Gould RJ, Polokoff MA, Freidman PA, et al. Disintegrins: a family of integrin inhibiting proteins from viper venom Proc Soc Exp Biol Med 1990;195:168-71.

53 Michie SA, Streeter PR, Bolt PA, et al. The human periph eral lymph node vascular adressin. Am f Pathol 1993;143 1688-98.

54 Nakamori S, Kameyama M, Imaoka S, et al. Increased expression of sialyl Lewis $\mathrm{x}$ antigen correlates with poor survival in patients with colorectal carcinoma: clinicopathological and immunohistochemical study. Cancer Res 1993;53:3632-7.

55 Gunthert U, Hofmann M, Rudy W, et al. A new variant of glycoprotein CD44 confers metastatic potential to rat carcinoma cells. Cell 1991;65:13-24.
56 Seiter S, Arch R, Komitowski D, et al. Prevention of tumour metastasis formation by anti-variant CD 44.7 Exp Med 1993;177:443-55.

57 Wielenga VJM, Heider K-H, Offerhaus GJA, et al. Expression of CD44 variant proteins in human colorectal cancer is related to tumour progression. Cancer Res 1993;53:4754-6.

58 Birchmeier W, Behrens J. Cadherin expression in carcinomas: role in the formation of cell junctions and the prevention of invasiveness. Biochem Biophys Acta 1994; 1198:11-26.

59 Rubinfeld B, Souza B, Albert I, et al. Association of the APC gene product with beta-catenin. Science 1993;262:1731-4.

60 Trofatter JA, MacColin MM, Rutter JL, et al. A novel moesin-, ezrin-, radixin-like gene is a candidate for the neurofibromatosis 2 tumor suppressor. Cell 1993;72:791800 .

61 Shibamoto S, Hayakawa M, Takeuchi K, et al. Tyrosine phosphorylation of beta-catenin and plakoglobin enhanced by hepatocyte growth factor and epidermal growth factor in human carcinoma cells. Cell Adhes Commun 1994;4:295305.

62 Behrens J, Vakaet L, Friis R, et al. Loss of epithelial differeniation and the gain of invasiveness correlates with tyrosine phosphorylation of the E-cadherin/ $\beta$-catenin complex in cells transformed with a temperature-sensitive v-src gene. $\mathcal{F}$ Cell Biol 1993;120:757-66.

63 Okazaki M, Takeshita S, Kawai S, et al. Molecular cloning and characterisation of OB-cadherin, a new member of the cadherin family expressed in osteoblasts. F Biol Chem 1994; 269:12092-8.

64 Katayama M, Hirai S, Kamihagi K, et al. Soluble E-cadherin fragments increased in circulation of cancer patients. $\mathrm{Br} F$ Cancer 1994;69:580-5.

65 Guo YJ, Liu G, Wang X, et al. Potential use of cd44 in serum as indicator of tumor burden and metastasis in patients with gastric or colon cancer. Cancer Res 1994;54:422-6.

66 Matsumura Y, Hanbury D, Smith J, et al. Non-invasive detection of malignancy by identification of unusual CD44 gene activity in exfoliated cancer cells. BMF 1994;308:61924 .

67 Sakuragi N, Nishiya M, Ikeda K, et al. Decreased E-cadherin expression in endometrial carcinoma is associated with tumor dedifferentiation and

68 Umbas R, Isaacs WB, Bringuier PP, et al. Decreased E-cadherin expression is associated with poor prognosis in E-cadherin expression is associated with poor prognosis in

69 Bracke ME, Charlier C, Bruyneel EA, et al. Tamoxifen restores the E-cadherin function in human breast cancer MCF-7/6 cells and suppresses their invasive phenotype. Cancer Res 1994;54:4607-9.

70 Lipponen P, Saarelainen E, Ji H, et al. Expression of E-cadherin (E-CD) as related to other prognostic factors and survival in breast cancer. F Pathol 1994;174:101-9.

71 Penno MB, August JT, Baylin SB, et al. Expression of CD44 in human lung tumors. Cancer Res 1994;54:1381-7.

72 Dall P, Heider KH, Hekele A, et al. Surface protein expression and messenger RNA-splicing analysis of CD44 in uterine cervical cancer and normal cervical epithelium. Cancer Res 1994;54:3337-41.

73 Culty M, Shizari M, Thompson EW, et al. Binding and degradation of hyaluronan by human breast cancer cell lines expressing different forms of CD44: correlation with invasive potential. F Cell Physiol 1994;160:275-86.

44 Mulder JW, Kruyt PM, Sewnath M, et al. Colorectal cancer prognosis and expression of exon-v6-containing CD44 proteins. Lancet 1994;344:1470-2.

75 Sawada R, Tsuoboi S, Fukuda M. Differential E-selectindependant efficiency in sublines of a human colon cancer exhibiting distinct metastatic potentials. F Biol Chem 1994; 269:1425-31. 